\title{
Impression of smoothness of a sound stream in relation to legato in musical performance
}

\author{
SONOKO KUWANO, SEIICHIRO NAMBA, and TERUO YAMASAKI \\ Osaka University, Osaka, Japan \\ and \\ KEIKO NISHIYAMA \\ Osaka, Japan
}

\begin{abstract}
Physically continuous sounds do not always produce the subjective impression of legato. In the present study, the effect of temporal factors on the impression of smoothness of a sound stream was systematically investigated in relation to the dynamic characteristics of hearing. The results showed that decaying sounds, successively presented, were perceived as being marginally connected when the sounds physically overlapped, while steady-state sounds were perceived as being marginally connected when they were physically separated. It was also shown that judgments by the subjects agreed quite well with the effect intended by the pianist when the passage was given in different temporal interpretations.
\end{abstract}

Since sounds convey information in a temporal stream, it is necessary to investigate the dynamic characteristics of hearing in order to examine information processing. Many experiments have been investigations of the dynamic characteristics of hearing, exploring such features as temporal masking, gap detection, detection of amplitude or frequency modulation, fluctuation strength, and temporal summation of loudness (e.g., Moore, 1989; Zwicker \& Fastl, 1990). Such experiments are usually conducted independently of each other, but when a series of sounds in a temporal stream is processed, as in the perception of speech or music, many aspects of hearing may work together and contribute to cognition. In the study of hearing there are two kinds of approach: to study each attribute or function independently, or to study the relation between attributes or functions and integrate them in relation to the phenomena in actual situations-that is, the verification of ecological validity. The present study follows the latter procedure. The factors determining the impression of legato in piano performance will be investigated, and the validity of a hypothesis arrived at on the basis of fundamental research will be examined.

A piano sound has a short attack and a fairly long decay-referred to as decaying sound in the present paper. The loudness of such sounds is roughly determined by their total energy (Kuwano, Namba, Miura, \& Tachibana, 1987; Namba, Kuwano, \& Kato, 1974), though close examination shows that the envelope pattern has a systematic, though small, effect on loudness. In an earlier ex-

Address correspondence to Sonoko Kuwano, College of General Education, Osaka University, 1-1 Machikaneyama, Toyonaka, Osaka, 560 Japan.

-Accepted by previous editor, Charles W. Eriksen periment (Namba, Kuwano, \& Kato, 1976) an intensity increment was added to steady-state sounds, and the temporal position of the increment was systematically varied, as shown in Figure 1. The duration of the pedestal portion was 350 or $700 \mathrm{msec}$ and that of the increment was one seventh that of the pedestal, as is shown in Figure 2. Even though the total energy of each example was equal, it was found that loudness varied according to the temporal position of the increment. When the increment was located at the onset of the sound, loudness was overestimated. This suggests that overshoot in the estimation of loudness may occur when the rise time of the sound is short. Underestimation occurred when the increment was located in the middle of the pedestal.

Overshoot at the onset of the sound was confirmed in an experiment on masking, in which steady-state sounds and decaying sounds were used as a masker (Namba, Hashimoto, \& Rice, 1987). Examples of these results are shown in Figures 3 and 4 . The experiment also confirmed that masking produced an aftereffect in the case of steadystate sounds, but not with decaying sounds when the fall time was relatively long. That is, when a sound with a relatively long decay was used as a masker, there was no effect of forward masking at the cessation of the sound.

On the basis of the results of these experiments (Namba et al., 1987; Namba et al., 1976), a schematic model of the dynamic characteristics of hearing has been proposed, which is shown in Figure 5. This is a basic model for a single sound. When successively presented sounds, such as musical performances, are considered in relation to this model, the following hypothesis can be made. Since there is no effect of forward masking with decaying sounds, such as piano sounds, it is necessary to shorten the intervals between sounds in order to give the impression of legato. Even when successive decaying sounds partially 


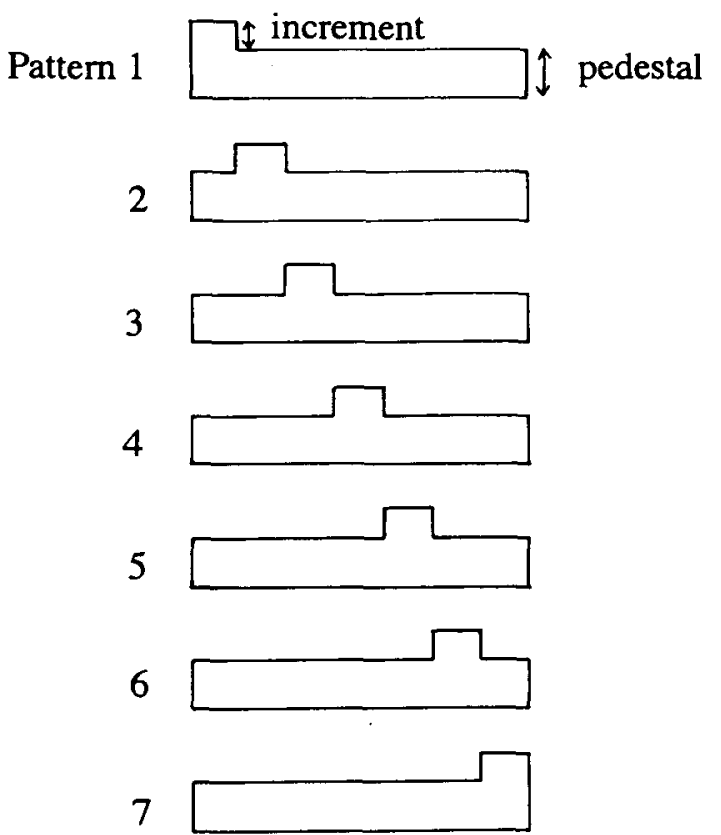

Figure 1. Stimulus patterns of non-steady-state sounds from a previous study. From "The Loudness of Sound With Intensity Increment," by S. Namba, S. Kuwano, and T. Kato, 1976, Japanese PSychological Research, 18, p. 64. Copyright 1976 by the Japanese Psychological Society. Reprinted by permission.

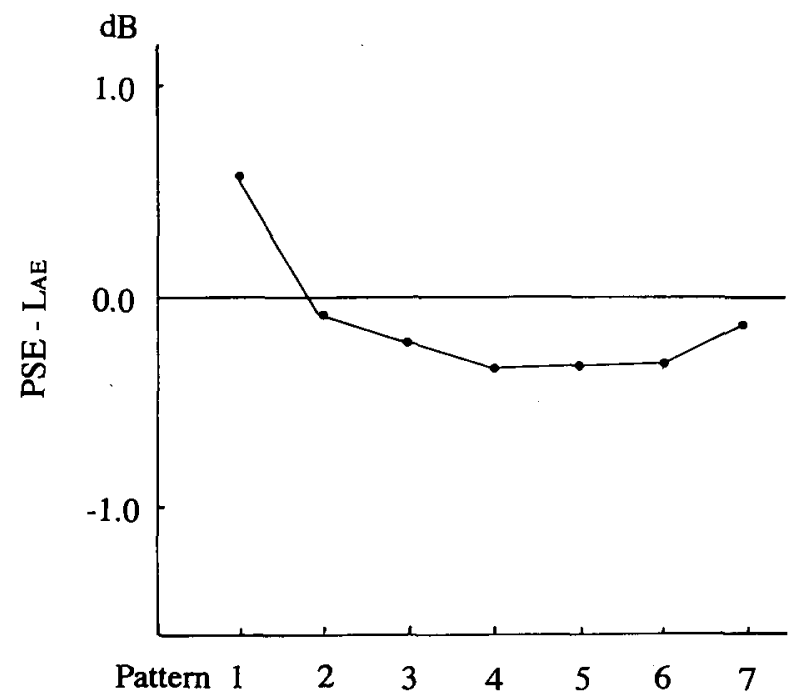

Figure 2. The loudness of non-steady-state sounds. It can be seen that loudness was overestimated when the increment was located at the onset of the stimulus. (LAE: sound exposure level.) From "The Loudness of Sound With Intensity Increment," by S. Namba, S. Kuwano, and T. Kato, 1976, Japanese Psychological Research, 18, p. 69. Copyright 1976 by the Japanese Psychological Society. (Only average data are plotted here from the original figure.) Adapted by permission.

overlap each other, they are probably not perceived as being impure. This is because the decaying portion of the preceding sound, which has little energy, may be effectively masked by the overshoot at the onset of the fol- lowing sound. This suggests that, in a piano performance, it would be better to overlap successive sounds with each other, though it would be necessary for the duration of overlapping to be short in order to produce the smooth impression of legato.

On the other hand, there is an aftereffect at the cessation of steady-state sounds, with the result that successively presented steady-state sounds may be perceived as being continuous, even though they are slightly separated. When two sounds of the same energy overlap, the preceding sound cannot be masked by the following sound. This may create the impression that the sounds are impure, especially when the two sounds are dissonant with each other. In order to produce the smooth impression of legato with steady-state sounds, it would be better to separate the sounds slightly, instead of overlapping them.

The present study was conducted to examine whether this hypothesis, derived from the model shown in Figure 5, agrees with our actual perception of legato in $\mathrm{mu}$ sical performance. The reason for choosing legato is that we were interested in clarifying the factors determining the impression of the smoothness of legato in relation to the dynamic characteristics of hearing, since it is important, but not easy, to achieve the pleasing effect of legato in musical performances.

\section{EXPERIMENT 1}

Legato is a musical term that means "smoothly continuous performance." However, there is no objective or physical definition of "smoothly continuous." In Experiment 1 , a short melody was performed in which decaying sounds and steady-state sounds were included by the systematic variation of the intervals between sounds. Subjects made judgments as to whether they perceived the sounds as "separated," "marginally connected," or "overlapping," and the results were examined to find whether the judgment for each temporal interval corresponded with what would be predicted from the model.

\section{Method}

Stimulus. In experiments, it is better to use the simplest stimulus practicable. If a series of sounds of the same pitch and duration is used, however, this may have the effect of a pulse train, which is different from that produced by music. On the other hand, if very complex sounds are used, it will be difficult to find the dominant factor in determining the impression of legato.

In Experiment 1, the first two bars of Pictures at an Exhibition, composed by Mussorgsky, were used as the stimulus; the score for this is shown in Figure 6. It is performed with single sounds (not chords) and has a simple structure. The musical intervals between neighboring sounds are from the second to the fourth, including consonant and dissonant intervals. The same piece was used in a previous study on the impression of musical performance (see Namba, Kuwano, Hatoh, \& Kato, 1991). Six different sounds occur in these two bars, and these were continuously generated by a music synthesizer (Yamaha DX-7) and recorded with a DAT (digital audio tape recorder; Sony DTC-55ES). The passage was performed by using the Programmable Sound Control System II (Namba \& Kuwano, 1984) to control the duration, time intervals, and temporal envelope patterns of the six sounds. The performances were recorded with a DAT (Sony DTC-1000ES). 

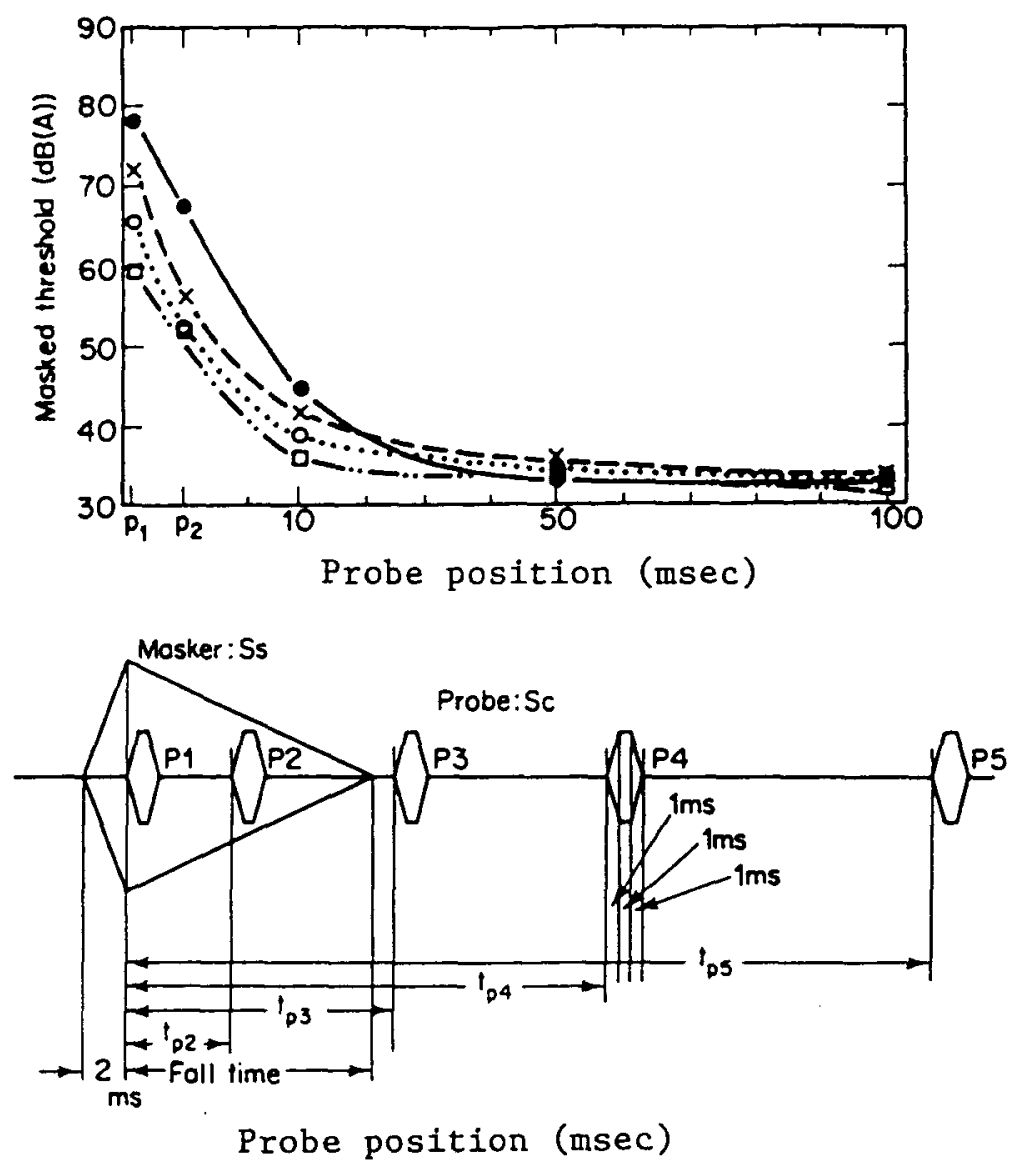

\begin{tabular}{crrrrr}
\hline Mosker foll time & $t_{p 1}$ & $i_{p 2}$ & $i_{p 3}$ & $i_{p 4}$ & $t_{p 5}$ \\
\hline 16 & 0 & 8 & 26 & 66 & 116 \\
64 & 0 & 32 & 74 & 114 & 164 \\
256 & 0 & 128 & 266 & 306 & 356 \\
512 & 0 & 256 & 522 & 562 & 612 \\
\hline
\end{tabular}

Figure 3. Temporal masking when the masker was a decaying sound. From "The Loudness of Decaying Impulsive Sounds," by S. Namba, T. Hashimoto, and C. G. Rice, 1987, Journal of Sound \& Vibration, 116, pp. 496-497. Copyright 1987 by Academic Press. Reprinted by permission.

Stimulus conditions. Two kinds of envelope pattern were used. One featured a short attack and a long decay, as with piano sound (a decaying sound); the pattern is shown in Figure 7a. The rise time was fixed at $10 \mathrm{msec}$, and the basic decay time was fixed at $590 \mathrm{msec}$ for a quarter note. The other was a steady-state sound with $10-\mathrm{msec}$ rise time and 10 -msec decay time. The basic duration of the steadystate portion was $\mathbf{5 8 0 ~ m s e c ~ f o r ~ a ~ q u a r t e r ~ n o t e ; ~ t h e ~ p a t t e r n ~ i s ~ s h o w n ~}$ in Figure $7 \mathrm{~b}$.

The time interval between the sounds was varied from $-150 \mathrm{msec}$ to $+300 \mathrm{msec}$ by 30 -msec steps for decaying sounds. As shown in Figure 8, the negative interval means that there is a silence between sounds, and the positive interval means that the sounds overlap with each other. For steady-state sounds, the time interval between the sounds was varied from $-180 \mathrm{msec}$ to $+180 \mathrm{msec}$ by 20 -msec steps. The total duration of the performance was fixed at $6.6 \mathrm{sec}$; that is, the rhythm pattern was kept constant so that each stimulus might be perceived as being a natural musical performance. Therefore, in order to control the time intervals between sounds, the duration of the decay time was varied for decaying sounds, and the duration of the steady-state portion was varied for steady-state sounds.

The time intervals between sounds and the range of the stimulus series for both decaying and steady-state sounds were determined on the basis of preliminary experiments, so that the stimulus range extended from a condition in which sounds were perceived as being clearly separated to a condition in which they were perceived as clearly overlapping. The sound-pressure level of each sound was 

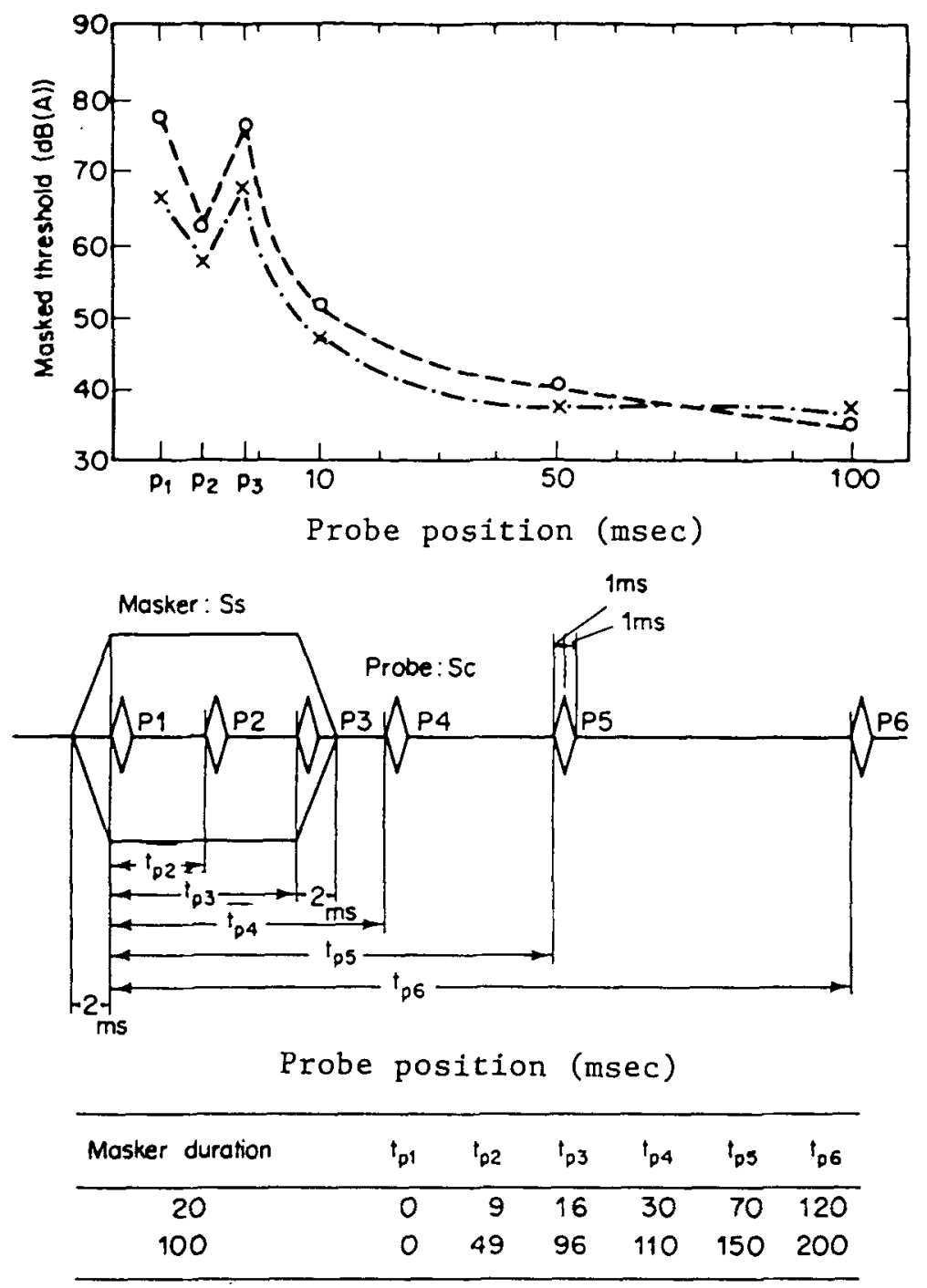

Figure 4. Temporal masking when the masker was a steady-state sound. From "The Loudness of Decaying Impulsive Sounds," by S. Namba, T. Hashimoto, and C. G. Rice, 1987, Journal of Sound \& Vibration, 116, pp. 498-499. Copyright 1987 by Academic Press. Reprinted by permission.

measured with an artificial ear (Brüel \& Kjaer 4153), a preamplifier (Brüel \& Kjaer 2660), and a measuring amplifier (Brüel \& Kjaer 2610), and was kept constant at $83 \mathrm{~dB}$ SPL.

Equipment. The stimulus was reproduced with a DAT (Sony DTC-1000ES) and presented to subjects through headphones (STAX SRD-X) diotically, to avoid the effect of room acoustics. The experiment was conducted in a soundproof room.

Procedure. The method of limit was used, since this was a preliminary experiment. The method of limit is easy for subjects to understand, and it can be conducted in a shorter time than the method of random presentation. A stimulus series consisted of performances with time intervals of sounds in ascending or descending order. The interval between performances was $3 \mathrm{sec}$. The subjects were instructed to judge each performance using three categories: "sounds overlapping," "sounds marginally connected," and "sounds separated." These categories were selected in order to find the condition in which the performance could be perceived as being "smooth." Experiment 1 consisted of two sessions. In each session, only one type of sound (decaying or steady-state) was used, and each subject responded to four ascending and four descending series with different starting points. Before each session, the subjects received training with one ascending series. If they did not understand the procedure, more training was given. Half of the subjects started the experiments with the session of decaying sounds, and the other half began with steady-state sounds.

Subjects. Twenty subjects participated: 8 females and 12 males, between 18 and 29 years of age, with normal hearing ability. None had had special training in music.

\section{Results and Discussion}

The results conformed with the model shown in Figure 5; they are shown in Table 1 . As can be seen, there is an interesting difference between the results with decay- 


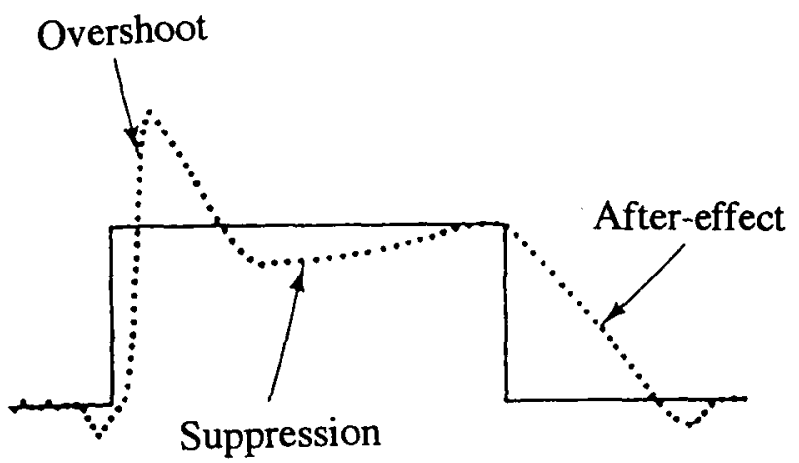

Figure 5. A model of dynamic characteristics of hearing. There is overshoot at the onset of the stimulus, suppression immediately after the onset, and aftereffect at the cessation of the stimulus. From "The Loudness of Sound With Intensity Increment," by S. Namba, S. Kuwano, and T. Kato, 1976, Japanese Psychological Research, 18, p. 70. Copyright 1976 by the Japanese Psychological Society. Reprinted by permission.

ing sounds and those with the steady-state sounds. The decaying sounds were judged to be marginally connected when the sounds overlapped each other by about $67 \mathrm{msec}$. This means that the sounds were not perceived as overlapping, even though they overlapped physically. On the other hand, as Table 1 shows, the steady-state sounds were judged to be marginally connected when the sounds were separated by about $20 \mathrm{msec}$. This result agrees well with the hypothesis based on the model. A $t$ test showed that there was a statistically significant difference between the results with the decaying sounds and those with the steady-state sounds $(t=9.815, p<.001)$.

A supplementary experiment was conducted in order to confirm the reliability of the results. The stimuli from Experiment 1 were used but the subjects judged each of them according to only two categories instead of three: "sounds separated" and "sounds overlapping." Eleven female and 9 male subjects, between 20 and 45 years of age with normal hearing ability, participated in the supplementary experiment. None had had special training in music. The average intervals at which the judgments changed from "sounds separated" to "sounds overlapping" (or vice versa) were $79.5 \mathrm{msec}$ for decaying sounds and $-10.6 \mathrm{msec}$ for steady-state sounds. Though there was little difference in the length of the intervals, these findings were similar to those in Experiment 1. A $t$ test showed that there was also a statistically significant difference between the results with decaying sounds and those with steady-state sounds $(t=3.630, p<.01)$. The results of Experiment 1 were confirmed by the supplementary experiment.

\section{EXPERIMENT 2}

In Experiment 1, a systematic relation was found between the time intervals between sounds and subjective impressions. These results agreed well with the hypothesis represented by the model in Figure 5. The stimuli used in Experiment 1 were artificial sounds generated by a music synthesizer, and their envelope patterns were simple. They were performed automatically, and the temporal conditions were exactly controlled; consequently, it was easy for the subjects to make judgments. However, the performances were unnatural compared with performances by actual pianists. It is questionable, however, whether pianists can systematically control temporal conditions in their performances.

Experiment 2 was designed to confirm the results of Experiment 1 when actual piano playing was used as the stimulus. Here, the questions arise of how precisely a pianist can control the duration of the overlapping of sounds and what the temporal condition is when the pianist intends the sounds to be perceived as being marginally connected.

\section{Method}

Procedure. The authors asked a pianist to play the same passage used in Experiment 1 in eight different temporal conditions, from "markedly separated"' to "extensively overlapping." An electronic piano (Roland HP-2700) was used so that physical measurement could be made easily, and the performances were recorded on a DAT (Sony DTC-1000ES) and stored in a computer through MIDI. Before playing, the pianist was instructed in the desired tempo of the performance by means of a metronome, and she played the passage according to the memorized tempo.

Analysis. The intervals between the touching of keys ( $t_{1}$ in Figure 9 , top) were directly measured by the MIDI information, which also included the duration and velocity of the touching of keys. Each sound was individually replayed by using a computer programmed with the MIDI information, and was measured using a waveform editor (CANOPUS Sound Master), as shown in Figure 9 (bottom). In the figure, " $A$ " is the point at which the pianist touched the $k e y$, and " $B$ " is the point at which sound-pressure level decreased $60 \mathrm{~dB}$ from the maximum level. The interval between $A$ and $B$ was regarded as the duration of a sound $\left(t_{2}\right)$. The difference between

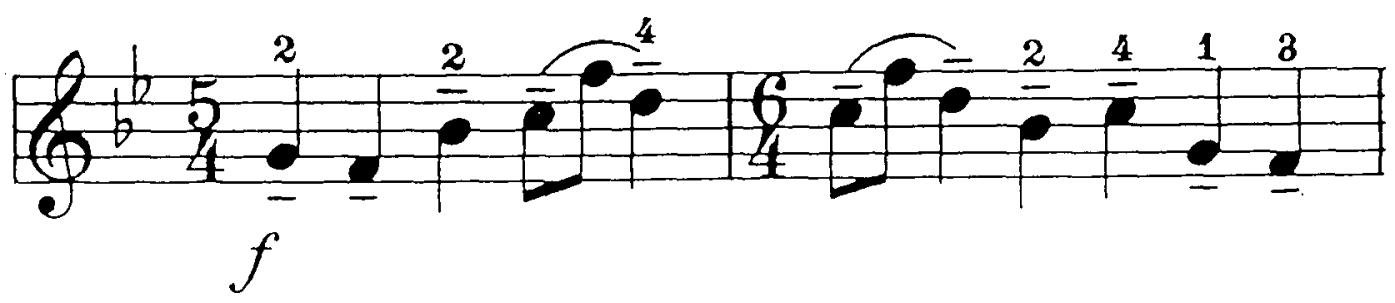

Figure 6. The score of the first two bars of Pictures at an Exhibition, composed by Mussorgsky, which was used in the experiments. 
(a)

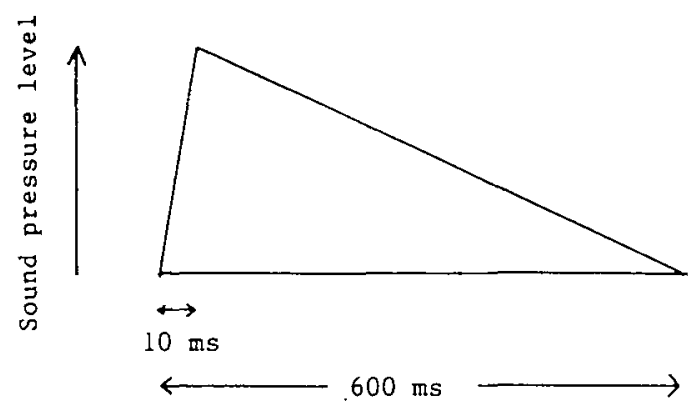

(b)

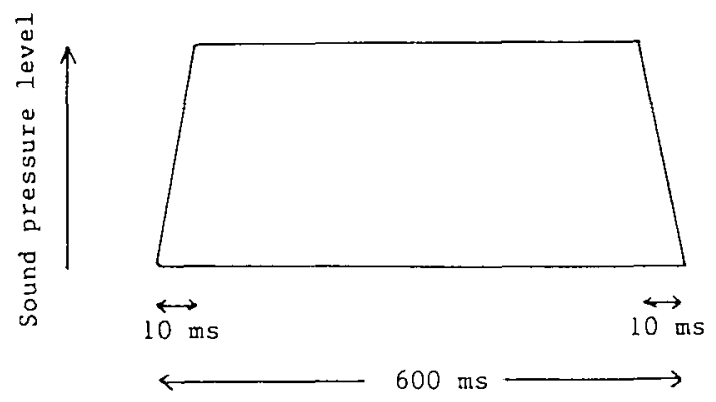

Figure 7. Temporal patterns of the stimuli used in the experiments: decaying sound (a) and steady-state sound (b).

the duration $\left(t_{2}\right)$ and the interval $\left(t_{1}\right)$ was regarded as the duration when the two sounds overlapped.

\section{Results and Discussion}

The results of analysis are shown in Figure 10. The bottom horizontal line indicates the average duration during which each note overlapped the following note, and the left vertical line indicates the temporal conditions that were intended by the pianist. The open circles and filled squares indicate the results with quarter notes and eighth notes, respectively. Except for extreme categories, the results for both notes agreed well.
A clear relation can be seen between the pianist's intention and the duration that was realized, which suggests that a pianist can control the duration of overlapping. The average duration of overlapping for the performance that the pianist intended to be "marginally connected" was about $240 \mathrm{msec}$. This overlapping is very extensivemuch longer than that found in Experiment 1.

In Experiment 1, artificial sounds with a simple envelope pattern were used, as is shown in Figure 7. In Experiment 2, however, the envelope pattern of electronic piano sounds, the timbre of which was similar to that of an actual piano, was complicated; the sound level

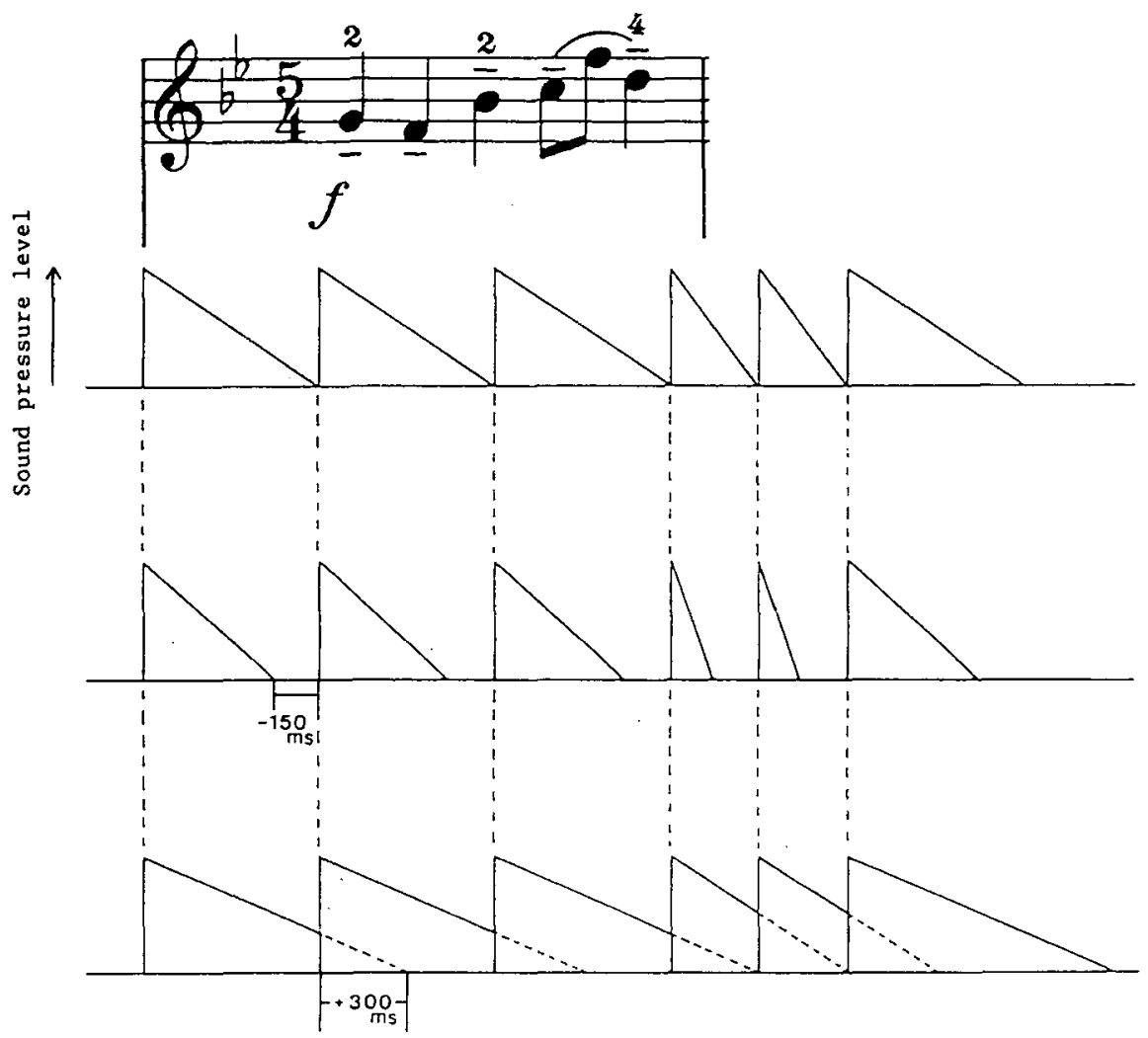

Figure 8. Temporal conditions used in the experiments. The intervals between sounds were varied from $-150 \mathrm{msec}$ to +300 msec by 30 -msec steps for decaying sounds, and from -180 msec to +180 msec by 20 -msec steps for steady-state sounds. A negative interval means that the sounds are physically separated, and a positive interval means that they physically overlap. 
Table 1

Results of Experiment 1

\begin{tabular}{ccc} 
& Decaying Sound & Steady-State Sound \\
\hline $\mathrm{A}$ & +30.0 & -56.0 \\
$\mathrm{~B}$ & +103.6 & +16.1 \\
$(\mathrm{~A}+\mathrm{B}) / 2$ & +66.8 & -19.9 \\
$\mathrm{C}$ & +62.8 & -12.2
\end{tabular}

Note $-\mathbf{A}=$ middle point between "sounds separated" and "sounds connected" judgments. B = middle point between "sounds connected" and "sounds overlapping" judgments. $\mathbf{C}=$ median of "sounds connected" judgments. The average values of $\mathrm{A}$ and $\mathrm{B}$ and the values of median (C) for both sounds approximate one another closely. All values are in milliseconds.

decreased while temporally fluctuating. The difference in envelope pattern may affect the impression of sound streams. It would be interesting to investigate the dynamic characteristics of hearing through an examination of the relation between the impression of sound as being either "smooth" or "interrupted" and the fine structure of the envelope pattern. The spectral components of the sounds were also different in Experiments 1 and 2. The effect of spectral components on the impression of sound as smooth or interrupted is also a subject that requires investigation.

\section{EXPERIMENT 3}

In Experiment 2 it was found that a pianist could control the impression of legato by changing the time intervals between sounds. The purpose of Experiment 3 was to examine whether a listener could discriminate the difference in performances with various durations of overlapping.

\section{Method}

Stimulus. The eight performances played by the pianist in Experiment 2 were used as stimuli.

Procedure. The eight performances were presented in random order. Subjects were asked to judge whether the sounds of each performance were "separated," "marginally connected," or "overlapping." After training, they judged each performance five times.

Subjects. Four females and 2 males, between 25 and 44 years of age with normal hearing ability, participated in the experiment.

\section{Results and Discussion}

The results are shown in Figure 11. The bottom horizontal line indicates the duration of overlapping in performance, and the left vertical line indicates the percentages of each judgment. It was found that the judgments agreed quite well with the category chosen by the pianist. The 50th percentile point for the "sounds overlapping" judgment was about $287 \mathrm{msec}$, and that for "sounds separated" was about $167 \mathrm{msec}$. The maximum percentage was found for the "sounds marginally connected" judgment when the duration of overlapping was about $241 \mathrm{msec}$. This result shows that there was good agree- ment between the performance of the pianist and the judgments by the listeners. A pianist can control the time interval between sounds in order to produce impressions from staccato to legato, and listeners can discriminate the intended impressions.

To confirm the results of Experiment 3, a supplementary experiment was conducted, using two categories. Eleven females and 9 males, between 20 and 45 years of age, participated. After training, the subjects made five judgments-either "separated" or "overlapping"-in each performance.

The results are shown in Figure 12. When two categories were used, the border (threshold) between the two categories (i.e., "sounds separated"' and "sounds overlapping") is clear. According to the subjects, however,
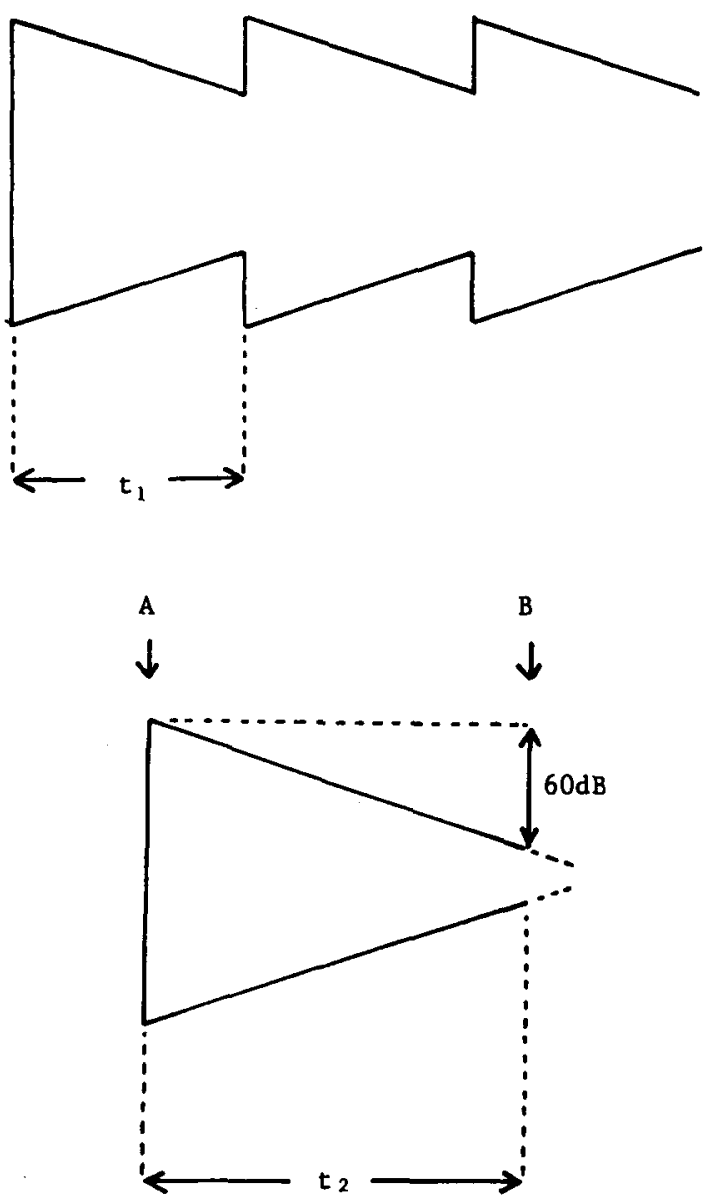

Figure 9. Schematic illustration of the duration measurement for overlapping sounds played on an electronic piano. Shown is the measurement of the interval between sounds on the basis of MIDI information (top) and the measurement of duration of each sound (bottom). The difference between $t_{2}$ and $t_{1}$ was regarded as the duration of overlapping. 


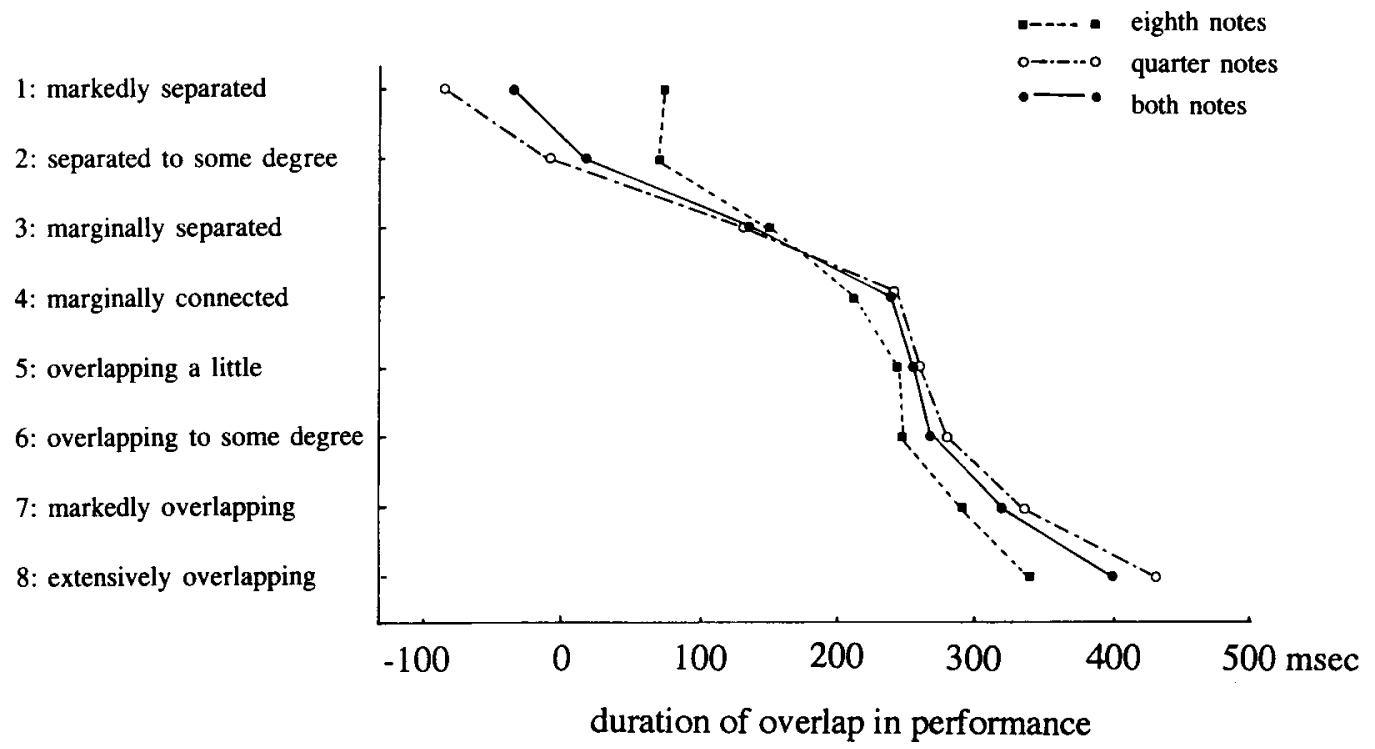

Figure 10. The relation between the average duration of overlapping of notes played by a pianist and the temporal conditions intended by the pianist. A clear relation can be seen between them.

it was easier for them to judge with three categories than with two. The "sounds separated" judgment showed a similar tendency in both experiments. Judgments in the category of "sounds marginally connected" in Experiment 3 seem to have been included in the "sounds overlapping" judgment in the supplementary experiment. There may be some range of intervals in which subjects will perceive the stimulus as "sounds marginally connected," and it is possible that the lower limen of the range is the threshold between "overlapping" and "separated" that was obtained in the supplementary experiment.
Though there may be other possible interpretations concerning the difference in threshold obtained in both experiments, it is nevertheless clear that the subjects consistently perceived the sounds that physically overlapped as being separated in the supplementary experiment.

\section{GENERAL DISCUSSION}

In the present experiments, it was clear that the impression of legato in musical performance was affected by the envelope patterns of the stimuli. When the "marginally

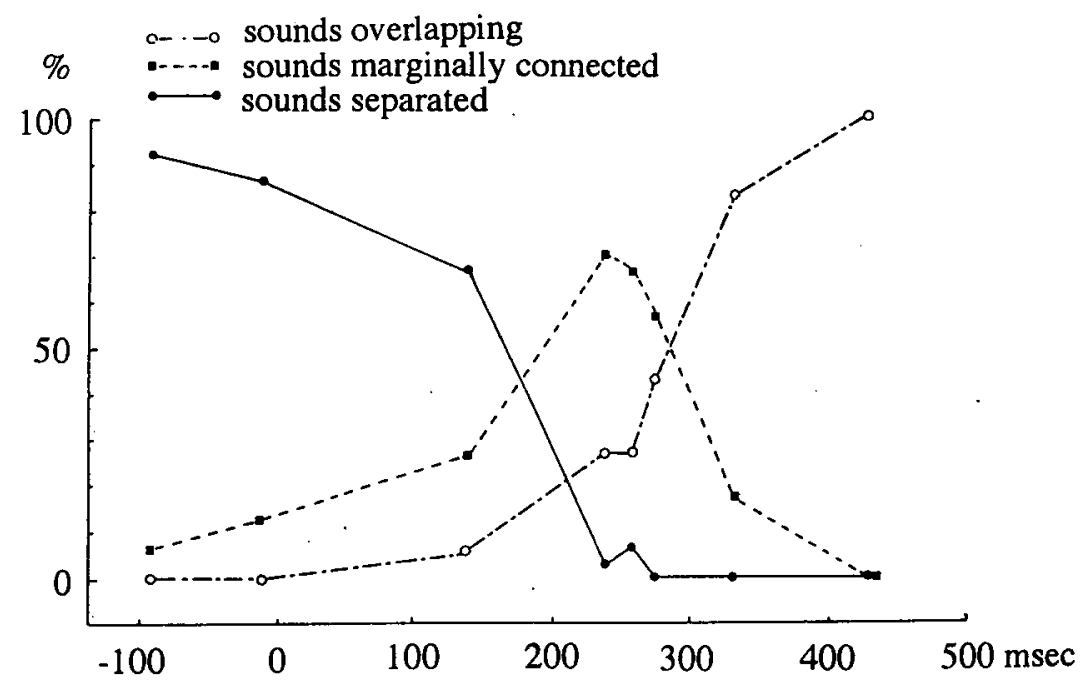

Figure 11. The relation between the duration of overlapping in performance and the percentages of each judgment. The judgments agree quite well with the category chosen by the pianist. 


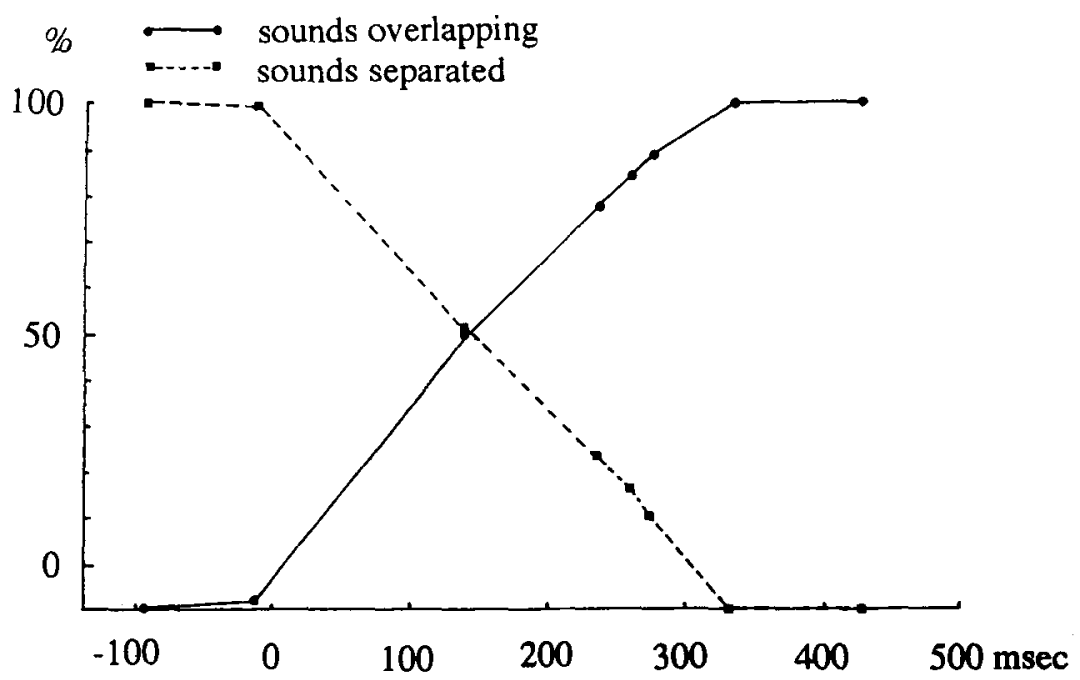

Figure 12. The results of the supplementary experiment, in which the performances were judged using two categories. The results were similar to those of Experiment 3.

connected" judgment is regarded as an index of the detection of a temporal gap, the threshold for steady-state sounds was smaller than that shown for pulse trains in previous studies (Buus \& Florentine, 1985). On the other hand, decaying sounds were perceived as being "marginally connected" even though the sounds overlapped. This is the phenomenon in which sounds are perceived as being separated even though they are physically overlapping. The opposite is true for steady-state soundsthey were perceived as being continuous even though they were physically separate. Both are examples of what may be called auditory illusion.

These results can be interpreted in the light of the dynamic characteristics of hearing, which are represented by the model in Figure 5. The envelope patterns of sounds have a great effect on the impression of continuity. Though the model in Figure 5 is only schematic and cannot predict the threshold for detecting a gap, it can be used to estimate the effect of envelope patterns. Since the dynamic characteristics of hearing are nonlinear, it is questionable whether the threshold for detecting a gap can be expressed with a simple constant-that is, whether the sounds are perceived as being continuous or separated may differ greatly, depending on the stimuli used.

One of the factors influencing gap detection is a fluctuation of the envelope pattern. For example, it is possible that when the pianist played an electronic piano in Experiment 2, the subtle fluctuation of the envelope pattern contributed to the high value of the threshold for the detection of a gap. The present experiments were designed to show that factors other than the time interval between sounds may influence the impression of legato. This inclusive approach is important for understanding perception in actual situations, where various factors interact with each other.
The purpose of the present experiments was to examine whether the basic model based on psychophysical experiments with single sounds can be applied to complex phenomena such as the impression of legato in musical performances. The closer the stimulus conditions are to actual situations, the more complex they become and the more difficult it is to find the determining factors. In the present experiments it became clear that envelope patterns influenced the impression of legato. However, other factors, such as frequency difference between sounds (consonant or dissonant), subtle fluctuation of amplitude, frequency of envelope patterns, and so forth, could not be examined systematically. Since the impression of legato is a matter of the successive overlapping of sounds, the frequency difference between sounds is a very important factor with regard to successive masking. The duration of each sound is also an important factor. These are areas that require future investigation.

\section{CONCLUSIONS}

When successively presented sounds were perceived as being marginally connected, it was found that the time interval was different according to the temporal patterns of the sounds-either physically overlapping (for decaying sounds) or physically separated (for steady-state sounds). This result could be arrived at by reference to the model of the dynamic characteristics of hearing, which is based on psychophysical experiments using single sounds.

It was found that the subjects' judgments agreed quite well with the pianist's category when she played the passage in different temporal conditions. A physical analysis of the performances showed that there was fairly extensive overlapping of sounds in the performance that was 
played with the intention that the sounds might be perceived as being marginally connected.

Further investigation using different melodies is being planned. It would also be of great interest to examine this matter in connection with hearing models.

\section{REFERENCES}

Buus, S., \& Florentine, M. (1985). Gap detection in normal and impaired listeners: The effect of level and frequency. In A. Michelsen (Ed.), Time resolution in auditory systems (pp. 159-179). Berlin: Springer-Verlag.

Kuwano, S., Namba, S., Miura, H., \& Tachibana, H. (1987). Evaluation of the loudness of impulsive sounds using sound exposure level based on the results of a round robin test in Japan. Journal of the Acoustical Society of Japan (E), 8, 241-247.

MOORE, B. C. J. (1989). An introduction to the psychology of hearing. London: Academic Press.

Namba, S., Hashimoto, T., \& Rice, C. G. (1987). The loudness of decaying impulsive sounds. Journal of Sound \& Vibration, 116, 491-507.

Namba, S., \& Kuwano, S. (1984). Effects of temporal structure of masker on temporal masking. Appendix: Measurement of simultaneous masking using PSCS II. Transactions of the Technical Committee on Hearing: The Acoustical Society of Japan, H-84-1, 1-6. (In Japanese)

Namba, S., Kuwano, S., Hatoh, T., \& Kato, M. (1991). Assessment of musical performance by using the method of continuous judgment by selected description. Music Perception, 8, 251-275.

Namba, S., Kuwano, S., \& Kato, T. (1974). The relation between loudness and rise time as a function of energy. Journal of the Acoustical Society of Japan, 30, 144-150. (In Japanese with English abstract)

Namba, S., Kuwano, S., \& Kato, T. (1976). The loudness of sound with intensity increment. Japanese Psychological Research, 18, 63-72.

Zwicker, E., \& FASTL, H. (1990). Psychoacoustics: Facts and models. Berlin: Springer-Verlag.

(Manuscript received April 22, 1992; revision accepted for publication January 12,1994 .) 